\title{
White Matter Changes, Duration of Hypertension, and Age Are Associated With Cerebral Microbleeds in Hypertensive Patients of Different Stages
}

\section{Changhu Liang}

Cheeloo College of Medicine, Shandong University

Jing Wang

Cheeloo College of Medicine, Shandong University

Cheng Liu

Cheeloo College of Medicine, Shandong University

Lingfei Guo ( $\nabla$ glfsci@163.com )

Cheeloo College of Medicine, Shandong University

\section{Research Article}

Keywords: T2*-weighted, magnetic resonance imaging, white matter changes, cerebral microbleeds, hypertension

Posted Date: December 16th, 2020

DOI: https://doi.org/10.21203/rs.3.rs-127567/v1

License: (c) This work is licensed under a Creative Commons Attribution 4.0 International License. Read Full License 


\section{Abstract}

Purpose We aimed to investigate risk factors for the presence and number of cerebral microbleeds (CMBs) in patients with different hypertension stages, with an emphasis on the relationship between white matter changes (WMCs) and CMBs.

Materials and methods Since 2016, participants aged 40 years or more have been evaluated for the presence of CMBs using enhanced 3D multi-echo GE T2*-weighted angiography (ESWAN) sequences. The Mann-Whitney U test and Pearson $\chi^{2}$ test were used to compare the clinical characteristics between the CMB and no-CMB groups of patients. Furthermore, we used Spearman's rank correlation analyses to examine the association between the degree of CMB severity and other important factors.

Results CMBs were detected in 110 (36.7\%) of 300 participants. Among patients with stage 2 hypertension, the majority also had CMBs. CMBs were positively correlated with age, hypertension stage, duration of hypertension, WMCs, and silent cerebral infarction. Patients with grade 3 WMCs were significantly more likely to have CMBs than those without WMCs; this association was true for patients with both stage 1 and stage 2 hypertension. In patients with stage 1 or stage 2 hypertension lasting longer than 20 years, the majority had CMBs.

Conclusions CMBs detected in hypertensive patients are more likely to occur in deep structures, and the grade of WMCs and duration of hypertension are more closely associated with the CMB degree than with age.

Synopsis: The grade of WMCs and duration of hypertension are more closely associated with the CMB degree than with age.

\section{Introduction}

Cerebral microbleeds (CMBs) are defined as focal signal intensity losses that are presumably secondary to ironcontaining hemosiderin residua of hemoglobin breakdown [1], which can be visualized using T2*-weighted gradientrecalled-echo (GRE) magnetic resonance imaging (MRI) and are associated with the presence and risk of stroke [2]. CMBs are affected by cerebral amyloid angiopathy (CAA) or hypertensive arteriopathy [3, 4].

White matter changes (WMCs) are defined as areas with high signal intensity that are visible using T2-weighted or fluidattenuated inversion recovery (FLAIR) MRI and as areas of low attenuation on CT images. The mechanisms by which WMCs develop are not fully understood and may include enlarged perivascular spaces; degeneration of myelin and axons with increased intracellular and extracellular water content; gliosis; and infarction [4-6].

Age, diagnosis with intracerebral hemorrhage $(\mathrm{ICH})$ or ischemic stroke, dementia, hypertension, and use of antithrombotic therapy have been associated with a higher risk of CMB detection using traditional 2D T2*-weighted GRE imaging.Although CMBs may occur with increased frequency in hypertensive patients [7, 8], we know little about their relationship with WMCs and the duration of hypertension for patients with different stages of hypertension. Some studies have reported WMCs in subjects displaying CMBs, but no study has primarily focused on the WMC-CMB relationship in patients with different stages of hypertension.

We performed an enhanced 3D multi-echo GE T2*-weighted angiography (ESWAN) sequence to detect CMBs and investigated the risk factors associated with the presence and number of CMBs among patients with different hypertension stages, emphasizing the relationship between WMCs and CMBs.

\section{Materials And Methods}




\section{Participants}

This study was approved by the institutional review board of Shandong Medical Imaging Research Institute Affiliated to Shandong University and all methods were carried out in accordance with relevant guidelines and regulations. All participants were informed of the detailed experimental procedures and signed informed consent forms. Between July 2016 and May 2018, all consecutive patients who were evaluated for hypertension were screened for participation in the present study. The inclusion criteria for this study were hypertensive patients over 40 years old. Patients must have undergone MRI examinations based on clinical symptoms suggestive of intracerebral hemorrhage (such as headache, migraine, tinnitus, insomnia, limb numbness, or transient ischemic attack symptoms), cognitive function disorders, lacunar infarction, or transient ischemic attack in conjunction with health-screening brain tests performed at our institute. Patients meeting these criteria were considered as potential subjects in this study. Patients were diagnosed according to the hypertension diagnosis standard. Patients were excluded from the study if conventional MRI revealed intracerebral hemorrhage, dementia, consciousness disorders, cerebral tumor, cerebral trauma, cavernous hemangioma, or arteriovenous malformation.

In total, 327 participants were eligible for our study; of those, 320 participants agreed to participate and gave informed consent. In 11 participants, imaging could not be completed due to physical problems; in 9 cases, motion artifacts prevented proper image assessment. In total, 300 hypertensive participants ( 160 males and 140 females) were included in the analysis. The average patient age was $61.70 \pm 10.31$ years (range: $40-85$ years). The average amount of time that passed since the patients had been diagnosed with hypertension was $17.00 \pm 8.10$ years (range: 1-43 years). Patients were diagnosed according to the hypertension diagnosis standard (Table 1).

Table 1

Classification of blood pressure for adults (JNC 7 Express)

\begin{tabular}{|lll|}
\hline Blood Pressure Classification & SBP $(\mathbf{m m H g})$ & DBP $(\mathbf{m m H g})$ \\
\hline Normal & $<120$ & and $<80$ \\
\hline Prehypertension & $120-139$ & or $80-89$ \\
\hline Stage 1 Hypertension & $140-159$ & or $90-99$ \\
\hline Stage 2 Hypertension & $>160$ & or $>100$ \\
\hline $\begin{array}{l}\text { SBP, systolic blood pressure; DBP, diastolic blood pressure; JNC 7 Express, the Seventh Report of the Joint National } \\
\text { Committee on Prevention, Detection, Evaluation, and Treatment of High Blood Pressure, U.S. National Institutes of }\end{array}$ \\
\hline
\end{tabular}

\section{MR Protocols}

Imaging was performed using a 3.0-T MRI scanner (General Electric Medical Systems, USA) with an eight-channel head coil. Two technologists performed all the MR imaging examinations according to a standardized conventional protocol. The parameters for the MR sequences with spin echo FLAIR T1-weighted images were as follows: repetition time (TR), $1977 \mathrm{~ms}$; echo time (TE), $27.7 \mathrm{~ms}$; and inversion time (TI), $860 \mathrm{~ms}$. The following parameters were used for fast spin echo T2-weighted images: TR, $3280 \mathrm{~ms}$ and TE, $106.9 \mathrm{~ms}$ and for T2 FLAIR: TR, $9002 \mathrm{~ms}$; TE, $155.7 \mathrm{~ms}$; and TI, $2250 \mathrm{~ms}$. The parameters for ESWAN were as follows: TR, $43.8 \mathrm{~ms}$; TE, $5.6 \mathrm{~ms}$; flip angle, $20^{\circ}$; bandwidth, $62.5 \mathrm{~Hz}$; slice thickness, $2 \mathrm{~mm}$; slice gap, $0 \mathrm{~mm}$; matrix, $480 \times 388$; field of view, $240 \times 240 \mathrm{~mm}^{2}$; and time of examination: approximately 6 minutes. 
The images were processed using ESWAN software in Functool according to the manufacturer's instructions. All images were transferred to an ADW4.3 workstation for ESWAN post-processing. As part of the reconstruction processes, varying phase shifts arising predominantly from background field heterogeneity and air-tissue interfaces were removed.

\section{Evaluation of Blood Pressure}

Conventional office blood pressure (BP) was measured at the hospital using sphygmomanometry (Korotkoff phases I and V) [9]. After at least 5 minutes of rest, three consecutive measurements were taken using the nondominant arm, with the participant seated, by the same trained investigator.

\section{Rating of Cerebral Microbleeds}

In this study, CMBs were defined as homogeneous round foci, $\leq 10 \mathrm{~mm}$ in diameter (no minimum size was specified), of low signal intensity on GRE T2*-weighted (or ESWAN) MRI [10] and excluding symmetrical low-signal intensity in the globus pallidus or empty flow in the distal-side branch cross at the cerebral artery branch. Vascular flow voids and basal ganglia mineralization were also excluded. The following seven regions were observed: the cortex, subcortical white matter, external capsule (EC)/internal capsule (IC), thalamus, basal ganglia grey matter, brainstem, and cerebellum. The cortex included subcortical regions touching the grey-white matter junction; the subcortical regions included the periventricular white matter and deep portions of the centrum semiovale; and basal ganglia grey matter included the caudate and lentiform nuclei. CMBs were classified by degree and assigned scores based on the total number of lesions as follows: 0 = absent, 1 = mild (1 to 2$), 2$ = moderate (3 through 10), and 3 = severe (>10) [11].

WMCs on MR images were defined as ill-defined hyperintensities $\geq 5 \mathrm{~mm}$ visible on both T2 and PD/FLAIR images and on CT as ill-defined and moderately hypodense areas $\geq 5 \mathrm{~mm}$ across. Lacunes were defined as well-defined areas of $2 \mathrm{~mm}$ with attenuation (on CT) or signal characteristics (on MRI) matching cerebrospinal fluid. If lesions with these characteristics were $\leq 2 \mathrm{~mm}$, they were considered perivascular spaces, except around the anterior commissure, where perivascular spaces can be large [12]. Lacunar infarction was defined as a small lesion (5 mm or larger in diameter) showing a low signal on T1-weighted images, a high signal on T2-weighted images, and a perilesional halo on FLAIR images [13]. The following rating scores were used to classify WMCs $(0-3)$ : Grade $0=$ no lesions (including symmetrical, well-defined caps or bands); Grade 1 = focal lesions; Grade 2 = beginning confluence of lesions; and Grade $3=$ diffuse involvement of the entire region, with or without the involvement of $U$ fibers.

The acquisition date and participant identification were removed from all images. ESWAN images were randomly allocated to one of four reviewers, who were blinded to the other sequence and to all clinical information. Reviewers independently rated the presence, location, and number of all CMBs. All studies with a potential microbleed were reviewed for confirmation by an experienced neuroradiologist.

\section{Statistical analysis}

All statistical analyses were performed using the SPSS 17.0 software. Twenty images of each sequence were read by both reviewers, with weighted $k$ values calculated as a measure of reproducibility; $k$ values less than 0.4 indicated poor agreement, values from 0.41 through 0.6 indicated moderate agreement, values from 0.61 through 0.8 indicated good agreement, and $\mathrm{k}$ values greater than 0.81 indicated excellent agreement [14]. To compare the clinical characteristics between the CMB and no-CMB groups, the Mann-Whitney $U$ test and Pearson's $\chi^{2}$ test (or Fisher's exact test) were used. To compare the differences in the WMC grade, duration of hypertension, and age between CMB and no-CMB groups at

different hypertension stages, the Pearson's $\chi^{2}$ test was used as appropriate. To detect the difference in the distribution 
of CMBs between hypertension stage 1 and 2 patients, we applied the Pearson's $\chi^{2}$ statistic or Fisher's exact test for categorical variables. Furthermore, using Spearman's rank correlation analyses, we examined the association between the degree of $\mathrm{CMBs}$ and WMC grade, duration of hypertension, and age of patients at different hypertension stages. A probability $(P)$-value $<0.05$ was considered significant.

\section{Results}

Interobserver reliabilities for the studies evaluated by both reviewers were good when analyzed both at the individual level (ESWAN, $\mathrm{k}=0.73$ ) and at the microbleed level (ESWAN, $\mathrm{k}=0.77)$.

The details of the demographic and MRI findings of the participants are listed in Table 2. CMBs were detected in 110 (36.7\%) of the 300 participants and in $75(68.2 \%)$ of participants over 60 years old. CMBs were similarly prevalent among men and women, with 59 (53.6\%) of 160 cases in men and 51 (46.4\%) of 140 cases in women $(P=0.09)$. 
Table 2

Clinical characteristics of the study participants according to the CMB status

\begin{tabular}{|c|c|c|c|c|}
\hline \multirow[b]{2}{*}{ Characteristics } & \multicolumn{4}{|c|}{ Cerebral microbleeds } \\
\hline & All (300) & CMBs (110) & $\begin{array}{l}\text { No CMBs } \\
(190)\end{array}$ & $P$ \\
\hline Age, years (IQR) & $61.70(40-85)$ & $70.3(46-85)$ & $65.4(40-80)$ & 0.001 \\
\hline \multicolumn{5}{|l|}{ Sex, n (\%) } \\
\hline Male & $160(53.3 \%)$ & $59(53.6 \%)$ & $101(53.2 \%)$ & 0.723 \\
\hline Female & $140(46.7 \%)$ & $51(46.4 \%)$ & $89(46.8)$ & 0.759 \\
\hline Education, years, median (IQR) & $9.3(0-19)$ & $9.1(0-17)$ & $10.4(3-19)$ & 0.565 \\
\hline Body mass index, kg/m², median (IQR) & $\begin{array}{l}22.3(19.1- \\
25.6)\end{array}$ & $\begin{array}{l}21.6(19.1- \\
25.2)\end{array}$ & $\begin{array}{l}24.6(19.4- \\
25.6)\end{array}$ & 0.124 \\
\hline \multicolumn{5}{|l|}{ Hypertension stage, n (\%) } \\
\hline Stage 1 & $154(51.3 \%)$ & $42(38.2 \%)$ & $112(58.9 \%)$ & 0.002 \\
\hline Stage 2 & $146(48.7 \%)$ & $68(61.8 \%)$ & $78(41.1 \%)$ & 0.004 \\
\hline Duration of hypertension, year, median (IQR) & $17.00(1-40)$ & $25(6-40)$ & $16(1-39)$ & 0.05 \\
\hline Diabetes mellitus, n (\%) & 34 & $15(13.6 \%)$ & $19(10 \%)$ & 0.076 \\
\hline Hyperlipidemia, n (\%) & 122 & $51(46.4 \%)$ & $71(37.3 \%)$ & 0.080 \\
\hline Coronary heart disease, $\mathrm{n}(\%)$ & 147 & $62(56.4 \%)$ & $85(44.7)$ & 0.050 \\
\hline Smoking, n (\%) & 90 & $34(30.9 \%)$ & $56(29.5 \%)$ & 0.524 \\
\hline Alcohol, units/week, median (IQR) & $0.16(0-13.0)$ & $0.15(0-13)$ & $0.14(0-12.5)$ & 0.789 \\
\hline Albumin, g/L, median (IQR) & $42.5(39-45)$ & $42.6(39-44.3)$ & $43.1(39-45)$ & 0.685 \\
\hline Fasting blood glucose, mmol/L, median (IQR) & $5.5(4.4-6.8)$ & $5.9(4.7-6.8)$ & $5.3(4.4-6.8)$ & 0.819 \\
\hline Total cholesterol, mmol/L, median (IQR) & $\begin{array}{l}5.11(4.01- \\
6.2)\end{array}$ & $5.6(4.53-6.2)$ & $\begin{array}{l}5.06(4.01- \\
5.9)\end{array}$ & 0.005 \\
\hline $\begin{array}{l}\text { High-density lipoprotein cholesterol, mmol/L, } \\
\text { median (IQR) }\end{array}$ & $\begin{array}{l}1.45(1.01- \\
1.91)\end{array}$ & $\begin{array}{l}1.40(1.01- \\
1.87)\end{array}$ & $\begin{array}{l}1.53(1.09- \\
1.91)\end{array}$ & 0.157 \\
\hline Triglyceride, mmol/L, median (IQR) & $\begin{array}{l}1.33(0.67- \\
2.03)\end{array}$ & $\begin{array}{l}1.27(0.67- \\
1.99)\end{array}$ & $\begin{array}{l}1.35(0.73- \\
2.03)\end{array}$ & 0.100 \\
\hline Creatinine, m mol/L, median (IQR) & $\begin{array}{l}58.7(43.6- \\
78.2)\end{array}$ & $\begin{array}{l}68.8(54.6- \\
78.2)\end{array}$ & $\begin{array}{l}51.5(43.6- \\
70.5)\end{array}$ & 0.030 \\
\hline Uric acid, m mol/L, median (IQR) & $\begin{array}{l}290(216- \\
450)\end{array}$ & $\begin{array}{l}357(218- \\
444)\end{array}$ & $310(216-450)$ & 0.05 \\
\hline WMC (grade 1-3) n (\%) & 275 & $108(98.2 \%)$ & $165(86.8)$ & 0.05 \\
\hline Silent cerebral infarction, n (\%) & 70 & $46(65.7 \%)$ & $24(12.6)$ & 0.001 \\
\hline
\end{tabular}

In patients with hypertension stage 1, the rate of cases with CMBs was significantly lower than the percentage of cases without CMBs $(P=0.002)$; in contrast, the rate of CMBs was significantly higher than that of cases without CMBs in 
patients with stage 2 hypertension $(P=0.004)$. There were significant differences in the average duration of hypertension between the participants with CMBs (25 years) and those without CMBs (16 years). In the patients diagnosed with silent cerebral infarction $(n=70)$, the percentage of the patients with CMBs was significantly higher than those without CMBs $(P=0.001)$.

Univariate analysis revealed that age, coronary heart disease, total cholesterol, creatinine, and uric acid were significantly associated with CMBs (Table 3). Simple correlation analysis explicitly showed that CMBs were positively correlated with age, hypertension stage, duration of hypertension, WMCs, and silent cerebral infarction, which showed the greatest clinical significance among these variables.

Table 3

Difference between the severity of WMC, duration, age and the presence of CMBs in patients with different stages of hypertension

\begin{tabular}{|c|c|c|c|c|c|c|c|}
\hline \multirow[b]{2}{*}{$\begin{array}{l}\text { Hypertension } \\
\text { Stage }\end{array}$} & & \multicolumn{2}{|c|}{ WMC grade } & \multicolumn{2}{|c|}{$\begin{array}{l}\text { Duration of hypertension } \\
\text { (years) }\end{array}$} & \multicolumn{2}{|l|}{ Age (years) } \\
\hline & & $0-2$ & 3 & $1-20$ & $>20$ & $40-60$ & $>60$ \\
\hline \multirow{2}{*}{$\begin{array}{l}\text { Stage } 1 \\
(n=154)\end{array}$} & $\begin{array}{l}\text { CMBs }(n= \\
42)\end{array}$ & $17(40.5 \%)$ & $25(59.5 \%)$ & $13(31.0 \%)$ & $29(69.0 \%)$ & $20(47.6 \%)$ & $22(52.4 \%)$ \\
\hline & $\begin{array}{l}\text { No CMBs }(\mathrm{n} \\
=112)\end{array}$ & $64(57.1 \%)$ & $48(42.9 \%)$ & $76(67.9 \%)$ & $36(32.1 \%)$ & $54(48.2)$ & $58(51.8 \%)$ \\
\hline$P$ & & 0.047 & 0.045 & 0.025 & 0.022 & 0.222 & 0.246 \\
\hline \multirow[t]{2}{*}{$\begin{array}{l}\text { Stage2 }(n= \\
146)\end{array}$} & $\begin{array}{l}\text { CMBs }(n= \\
68)\end{array}$ & $22(32.4 \%)$ & $46(67.6 \%)$ & $21(30.9 \%)$ & $47(69.1 \%)$ & $19(27.9 \%)$ & $49(72.1 \%)$ \\
\hline & $\begin{array}{l}\text { No CMBs }(n \\
=78)\end{array}$ & $40(51.3 \%)$ & $38(48.7 \%)$ & $45(57.7 \%)$ & $33(42.3 \%)$ & $30(38.5 \%)$ & $48(61.5 \%)$ \\
\hline$P$ & & 0.031 & 0.041 & 0.038 & 0.003 & 0.125 & 0.094 \\
\hline
\end{tabular}

Furthermore, we compared the differences between the severity of WMCs, duration of hypertension, age, and presence of CMBs in patients with different stages of hypertension (Table 3).

We divided the hypertensive patients into two groups according to their hypertension stage (1 or 2). In patients with grade three WMCs, the percentage of the patients with CMBs was significantly higher than those without CMBs $(P<0.05)$ in patients with both stage 1 and stage 2 hypertension. The participants of the present study had hypertension for an average of $17.00 \pm 8.10$ years (range: $1-43$ years); therefore, we divided them into a group with hypertension $\leq 20$ years and a group with hypertension $>20$ years. In the $>20$ years group, the percentage of patients with CMBs was significantly higher than those without CMBs for both stage 1 and stage 2 hypertensive patients $(P<0.05)$. When comparing middleaged and elderly patients, there were no significant differences in the percentage of patients with CMBs and those without CMBs $(P>0.05)$.

We examined the association between the degree $(0-3)$ of CMBs and the grade of WMCs $(0-3)$, duration of hypertension (1-10 years, 11-20 years, $21-30$ years, 31-40 years, $>40$ years), and age (40-50 years, 51-60 years, 6170 years, 71-80 years, > 80 years) among different hypertension stages using Spearman's rank correlation analyses (Table 4). In hypertension stage 2 patients, we found that the correlation between the duration of hypertension and both the degree of CMBs and grade of WMCs was highly positive $(r=0.712, P=0.003$ and $r=0.664, P=0.034$, respectively) (Fig. 1. a, b; Fig. 2. a, b). In stage 1 hypertensive patients, we found no correlation between these characteristics. In all 
participants, the correlation between the degree of CMBs and age was moderately positive in those with hypertension stage 1 or 2.

Table 4

Correlation data between the degree of CMBs and the grade of WMC, duration of hypertension, and age in patients with different stages of hypertension

\begin{tabular}{|c|c|c|c|c|}
\hline Hypertension stage & & WMC grade $(0-3)$ & Duration of hypertension (1-40 years) & Age (40-85 years) \\
\hline \multirow[t]{2}{*}{ Stage $1(n=42)$} & $\mathrm{r}$ & 0.396 & 0.297 & 0.423 \\
\hline & $P$ & 0.09 & 0.154 & 0.050 \\
\hline \multirow[t]{2}{*}{ Stage $2(n=68)$} & r & 0.712 & 0.664 & 0.435 \\
\hline & $\mathrm{P}$ & 0.003 & 0.034 & 0.05 \\
\hline
\end{tabular}

In the participants with CMBs and hypertension stage 1 or 2, CMBs were most commonly found in the basal ganglia grey matter and subcortical white matter. Additionally, the number of CMBs in the basal ganglia grey matter and brainstem was higher in patients with hypertension stage 2 than in patients with hypertension stage $1(P<0.05)($ Table 5$)$.

Table 5

Difference of CMB distribution by brain location in patients with different stages of hypertension

\begin{tabular}{|c|c|c|c|c|c|c|c|}
\hline \multirow{2}{*}{$\begin{array}{l}\text { Hypertension } \\
\text { stage }\end{array}$} & \multicolumn{7}{|l|}{ Location } \\
\hline & Cortex & $\begin{array}{l}\text { Subcortical } \\
\text { white } \\
\text { matter }\end{array}$ & $\begin{array}{l}\text { External } \\
\text { capsule/Internal } \\
\text { capsule }\end{array}$ & $\begin{array}{l}\text { Basal } \\
\text { ganglia } \\
\text { grey } \\
\text { matter }\end{array}$ & Thalamus & Brainstem & Cerebellum \\
\hline \multicolumn{8}{|l|}{$\begin{array}{l}\text { Total }(n= \\
300) \wedge\end{array}$} \\
\hline $\begin{array}{l}\text { Stage } 1(n= \\
154)\end{array}$ & $28(18.2 \%)$ & $77(50.0 \%)$ & $49(31.8 \%)$ & $76(49.3 \%)$ & $31(20.1 \%)$ & $10(.6 .5 \%)$ & $11(7.1 \%)$ \\
\hline $\begin{array}{l}\text { Stage } 2(n= \\
146)\end{array}$ & $30(20.5 \%)$ & 76(52.1\%) & $51(34.9 \%)$ & $90(61.6 \%)$ & $33(22.6 \%)$ & 17(11.6\%) & $10(6.8 \%)$ \\
\hline$P$ & $>0.05$ & $>0.05$ & $>0.05$ & $<0.05$ & $>0.05$ & $<0.05$ & $>0.05$ \\
\hline
\end{tabular}

\section{Discussion}

CMBs represent hemosiderin deposits that can remain in macrophages for years following a microhemorrhage.

Histopathologic studies have shown that $\mathrm{CMBs}$ are affected by moderate to severe fibro-hyalinosis and arteriolosclerosis [15]. Although the predictive value of CMBs for stroke prognosis has not been widely accepted, several clinical studies have suggested that CMBs might be associated with an increased risk of recurrent stroke [16, 17].

In recent years, there has been growing interest in determining the clinical significance of CMBs. One approach to interpreting $\mathrm{CMBs}$ is to regard them as a marker of accompanying vascular pathological changes. Histopathological analyses of the vessels associated with CMBs have generally identified the following two types of vascular pathological changes: hypertensive vasculopathy and CAA, which are distinguishable according to the location of the CMB. In the 
past year, a new scan study suggested that microbleeds visible using T2*-weighted MRI were a possible marker of both CAA and hypertensive vasculopathy progression [18].

Hypertension is a major risk factor associated with blood-brain barrier leakage of plasma components and may be a cause of WMCs and lacunar stroke [19]. Similarly, high BP during the day and at night, as well as a higher cumulative 24hour BP load, may induce structural changes in the brain microvasculature, causing subsequent extravasation of blood.

Recent studies have suggested that CMBs are frequently found in hypertensive patients lacking a history of cerebrovascular disease and are independently associated with higher daytime and nighttime BP levels [20]. The present study has demonstrated that, in this population, the prevalence of CMBs was $36.7 \%$. The prevalence of CMBs in our hypertensive cohort is approximately six times higher than that reported in the general population [21] and is approximately two times higher than patients without a history of cerebrovascular disease; these results are consistent with the incidence of CMBs in Chinese hypertensive patients. Importantly, the CMB count was not influenced by the history of cerebral tumor, cerebral trauma, encephalic angioma, or arteriovenous malformation because patients with these diseases were excluded from our study.

The prevalence of CMBs has been investigated in various populations. In elderly subjects lacking cerebrovascular diseases, the CMB prevalence is between $5 \%$ and $6 \%$ [22]; Tsushima et al. reported that the CMB incidence was $3.1 \%$ in 450 neurologically healthy Japanese adults. In addition, one study reported that CMB prevalence was closely related to hypertension and heavy cigarette smoking [23]. The frequency of CMBs in patients with stroke and healthy older subjects reported in our study is consistent with previously reported results [24]. The very high frequencies of stroke reported in some previous studies (e.g., 78\%) may reflect patient selection bias, with experiments only including subjects with hemorrhagic stroke. The prevalence of CMBs in our study population seems to be higher than these previous studies, which is attributable to hypertension in our subjects and/or higher accuracy in detecting CMBs through the use of a high-field system (3T MRI) [25, 26].

The ESWAN sequence is a new susceptibility-weighted imaging sequence that uses images of multiple magnitudes with different echo times to generate images. This technique avoids the complex post-processing required by other SWI approaches and may be useful in differentiating between hemosiderin and calcium [27, 28].

In our participants, we found an association between CMBs and age, hypertension stage, duration of hypertension, silent cerebral infarction, WMCs, coronary heart disease, total cholesterol, creatinine, and uric acid. The strengths of the present study include analyses of potential confounders such as age, duration of hypertension, and WMCs. Earlier studies have highlighted the relationship between $\mathrm{CMBs}$ and some conventional factors (e.g., gender, age, BP, smoking, white matter lesions, and lacunar infarction visible using MRI) [24, 29]; however, these studies only evaluated factors for ischemic or hemorrhagic diseases. To address this issue, we divided our participants into 2 groups based on their hypertension stage, allowing us to evaluate whether there is a significant correlation between the incidence of CMBs and conventional risk factors, including age, WMCs, and duration of hypertension.

The distributions of low-grade and high-grade WMCs differed between patients with different stages of hypertension. In patients with stage 1 hypertension, the rate of grade 3 or grade $0-2$ CMBs was no higher than the rate without CMBs. In contrast, the rate of CMBs was higher in patients with higher hypertension stage. When comparing the duration of hypertension between patients with $\mathrm{CMBs}$ and those without, the same result was found. These results suggest that the risk of $\mathrm{CMBs}$ is much higher in patients with higher $\mathrm{BP}$ levels, regardless of the hypertension duration or the degree of WMCs. Leon et al. performed a similar study [20] and concluded that CMBs were frequently found in hypertensive patients without a history of cerebrovascular disease and were independently associated with higher daytime and nighttime BP levels. The rate of CMBs in patients with stage 1 hypertension was no different between those who are middle-aged and older; the same held true for patients with stage 2 hypertension. 
In our study, to facilitate research classification, we divided the patients into two groups according to the JNC 7, which suggests that all people with hypertension (stages 1 and 2) should be treated. We chose to emphasize the relationship between age, duration of hypertension, and degree of WMCs and CMBs because these risk factors are easy to determine through interviews and the evaluation of MR or CT images taken during diagnosis or treatment. By preliminarily assessing the risk of CMBs in hypertensive patients on the basis of these factors, the radiologist targeted some patients for T2*-weighted sequence MRI, and the neurologist was able to consider the existence of CMBs in stage 2 hypertensive patients during treatment.

In the patients with stage 1 hypertension, a higher degree of CMBs was associated with some risk factors, including the WMC grade, duration of hypertension, and age, but the correlations between these factors and CMBs were not strong. The results suggest that there were fewer CMBs in patients with lower BP, which may indirectly explain how these factors influence small arteries. In the stage 2 hypertensive patients, the correlations between the risk factors and the degree of CMBs was stronger than in stage 1 patients. These factors may have a larger effect on the presence of CMBs, but the relationship between age and CMBs did not change much compared with the relationship in stage 1 hypertensive patients.

Additionally, we confirmed a close association between the number of CMBs, the severity of WMCs, and the duration of hypertension. Our results suggest that the risk associated with age was confounded by the risk associated with WMCs and that age had a weaker correlation with CMBs than both WMCs and the duration of hypertension. Age has been reported as one of the most important risk factors for not only CMBs but also high-grade WMCs [30, 31]. In these studies, advanced WMCs were assessed under similar conditions, consisting of extensive white matter damage from cerebral small vessel diseases such as microangiopathy and amyloid angiopathy.

We adopted the Wahlund scale, which has fewer grades, for evaluating the grade of WMCs. The scale was developed by the European Task Force on Age-Related White Matter Changes (ARWMC), which is a new scale applicable to both CT and MRI.

The possibility that all diffuse CMBs are the result of hypertensive microangiopathies must be considered. Indeed, chronic hypertension can influence the development of hypertensive microangiopathies anywhere in the brain [32], but CAA is typically found in cortical or leptomeningeal regions and is not necessarily related to hypertension [33]. Histopathologic analyse of CMBs that are visible using MRI have shown that CMBs reflect widespread involvement of the arterioles and are caused by hypertension, amyloid deposition, or both pathogenetic mechanisms. Thus, both pathogeneses might be involved in diffuse CMBs.

We found that CMBs occurring in deep structures were more common than those found in the lobar hemispheres of our participants. There were significantly more CMBs in the basal ganglia of hypertension stage 2 patients than in stage 1 patients. A similar result was true for the brainstem. These results suggest that CMBs in the brainstem should be a consideration when treating patients with stage 2 hypertension. The previous research showed that CMBs occurred more frequently in deep structures than in lobe [34], which is consistent with our study. The lobar hemispheres are common sites for hypertensive cerebral hemorrhages. Accordingly, these results suggest that modifiable risk factors such as high $\mathrm{BP}$ can be associated with CMBs.

The present study has several limitations, including the cross-sectional design and relatively small number of participants with CMBs. Although the group size was sufficient to perform the analyses, additional exploratory adjustments need to be interpreted within the context of their statistical limitations. Our findings require confirmation in longitudinal and adequately powered studies. Another limitation is the lack of a local, preferably community-based, control population. Such a control group would have enabled us to assess whether the prevalence of CMBs in our hypertensive cohort was higher than that in previous studies. 
Our study showed that CMBs are a frequent finding in patients. Moreover, the data suggest that participants with stage 2 hypertension are more likely to display CMBs on MRI than participants with stage 1 hypertension. In contrast to the general belief that CMBs are clinically silent, recent reports have associated them with cognitive impairment [35] and an increased risk of stroke recurrence [17], which illustrates the potential clinical relevance of these small lesions. We postulate that CMBs should be considered an independent marker of hypertensive target organ damage in the brain. Our findings require confirmation in adequately powered and preferably long-term follow-up studies, which should also address the role of CMBs in risk estimation and the prevention of both future strokes and impaired brain function.

\section{Conclusion}

In conclusion, the CMBs observed in hypertensive patients occurred more frequently in deep structures, and both the WMC grade and duration of hypertension have a closer association with the degree of CMBs than age. These results should be fully considered during clinical treatment. CMBs in hypertensive patients were more common in deep structures, and both the WMC grade and duration of hypertension were more closely associated with the degree of CMBs than age.

\section{Declarations}

\section{Conflicts of interest}

The authors declare no competing interests. Changhu Liang and Lingfei Guo wrote the main manuscript text. Jing Wang prepared Figures 1-4. Cheng Liu prepared the clinical data and imaging data. Lingfei Guo revised the main manuscript text. All authors reviewed the manuscript.

\section{Grant support}

This work was supported by grants from National Natural Science Foundation of China (81800840), Technology Development Plan of Jinan (201301049, 201602206, 201907052), Medical and Health Science and Technology Development Project of Shandong Province (2016WS0529) and Funding for Study Abroad Program by Shandong Province (No.201803059).

\section{References}

1. Schrag M, McAuley G, Pomakian J, Jiffry A, Tung S, Mueller C, Vinters HV, Haacke EM, Holshouser B, Kido D, Kirsch WM. Correlation of hypointensities in susceptibility-weighted images to tissue histology in dementia patients with cerebral amyloid angiopathy: a postmortem MRI study. Acta Neuropathol. 2010;119(3):291-302.

2. Offenbacher H, Fazekas F, Schmidt R, Koch M, Fazekas G, Kapeller P. MR of cerebral abnormalities concomitant with primary intracerebral hematomas. AJNR Am J Neuroradiol. 1996;17(3):573-8.

3. Fazekas F, Kleinert R, Roob G, Kleinert G, Kapeller P, Schmidt R, Hartung HP. Histopathologic analysis of foci of signal loss on gradient-echo T2*-weighted MR images in patients with spontaneous intracerebral hemorrhage: evidence of microangiopathy-related microbleeds. AJNR Am J Neuroradiol. 1999;20(4):637-42.

4. Vernooij MW, van der Lugt A, Ikram MA, Wielopolski PA, Niessen WJ, Hofman A, Krestin GP, Breteler MM. Prevalence and risk factors of cerebral microbleeds: the Rotterdam Scan Study. Neurology. 2008;70(14):1208-14. Epub 2008/04/02.

5. Naka H, Nomura E, Takahashi T, Wakabayashi S, Mimori Y, Kajikawa H, Kohriyama T, Matsumoto M. Combinations of the presence or absence of cerebral microbleeds and advanced white matter hyperintensity as predictors of subsequent stroke types. AJNR Am J Neuroradiol. 2006;27(4):830-5. 
6. van Es AC, van der Grond J, de Craen AJ, Admiraal-Behloul F, Blauw GJ, van Buchem MA. Risk factors for cerebral microbleeds in the elderly. Cerebrovasc Dis. 2008;26(4):397-403.

7. Koennecke HC. Cerebral microbleeds on MRI: prevalence, associations, and potential clinical implications. Neurology. 2006;66(2):165-71.

8. Cordonnier C, Al-Shahi Salman R, Wardlaw J. Spontaneous brain microbleeds: systematic review, subgroup analyses and standards for study design and reporting. Brain. 2007;130(Pt 8):1988-2003.

9. O'Brien E, Asmar R, Beilin L, Imai Y, Mallion JM, Mancia G, Mengden T, Myers M, Padfield P, Palatini P, Parati G, Pickering T, Redon J, Staessen J, Stergiou G, Verdecchia P. European Society of Hypertension recommendations for conventional, ambulatory and home blood pressure measurement. Journal of hypertension. 2003;21(5):821-48.

10. Cordonnier C, Potter GM, Jackson CA, Doubal F, Keir S, Sudlow CL, Wardlaw JM, Al-Shahi Salman R. improving interrater agreement about brain microbleeds: development of the Brain Observer MicroBleed Scale (BOMBS). Stroke. 2009;40(1):94-9.

11. Lee SH, Bae HJ, Yoon BW, Kim H, Kim DE, Roh JK. Low concentration of serum total cholesterol is associated with multifocal signal loss lesions on gradient-echo magnetic resonance imaging: analysis of risk factors for multifocal signal loss lesions. Stroke. 2002;33(12):2845-9.

12. Wahlund LO, Barkhof F, Fazekas F, Bronge L, Augustin M, Sjogren M, Wallin A, Ader H, Leys D, Pantoni L, Pasquier F, Erkinjuntti T, Scheltens P. A new rating scale for age-related white matter changes applicable to MRI and CT. Stroke. 2001;32(6):1318-22.

13. Koga H, Takashima Y, Murakawa R, Uchino A, Yuzuriha T, Yao H. Cognitive consequences of multiple lacunes and leukoaraiosis as vascular cognitive impairment in community-dwelling elderly individuals. Journal of stroke and cerebrovascular diseases. 2009;18(1):32-7.

14. Altman DG. Practical statistics for medical research. 1st ed. London ; New York: Chapman and Hall; $1991 .$.

15. Tanaka A, Ueno Y, Nakayama Y, Takano K, Takebayashi S. Small chronic hemorrhages and ischemic lesions in association with spontaneous intracerebral hematomas. Stroke. 1999;30(8):1637-42.

16. Fan YH, Zhang L, Lam WW, Mok VC, Wong KS. Cerebral microbleeds as a risk factor for subsequent intracerebral hemorrhages among patients with acute ischemic stroke. Stroke. 2003;34(10):2459-62.

17. Boulanger JM, Coutts SB, Eliasziw M, Gagnon AJ, Simon JE, Subramaniam S, Sohn CH, Scott J, Demchuk AM. Cerebral microhemorrhages predict new disabling or fatal strokes in patients with acute ischemic stroke or transient ischemic attack. Stroke. 2006;37(3):911-4.

18. Poels MM, Ikram MA, van der Lugt A, Hofman A, Krestin GP, Breteler MM, Vernooij MW. Incidence of cerebral microbleeds in the general population: the Rotterdam Scan Study. Stroke. 2011;42(3):656-61.

19. Chen YF, Chang YY, Liu JS, Lui CC, Kao YF, Lan MY. Association between cerebral microbleeds and prior primary intracerebral hemorrhage in ischemic stroke patients. Clinical neurology and neurosurgery. 2008;110(10):988-91.

20. Henskens LH, van Oostenbrugge RJ, Kroon AA, de Leeuw PW, Lodder J. Brain microbleeds are associated with ambulatory blood pressure levels in a hypertensive population. Hypertension. 2008;51(1):62-8.

21. Bokura H, Saika R, Yamaguchi T, Nagai A, Oguro H, Kobayashi S, Yamaguchi S. Microbleeds are associated with subsequent hemorrhagic and ischemic stroke in healthy elderly individuals. Stroke. 2011;42(7):1867-71.

22. Nishikawa T, Ueba T, Kajiwara M, Fujisawa I, Miyamatsu N, Yamashita K. Cerebral microbleeds predict first-ever symptomatic cerebrovascular events. Clinical neurology and neurosurgery. 2009;111(10):825-8.

23. Tsushima Y, Tanizaki Y, Aoki J, Endo K. MR detection of microhemorrhages in neurologically healthy adults. Neuroradiology. 2002;44(1):31-6.

24. Viswanathan A, Chabriat H. Cerebral microhemorrhage. Stroke. 2006;37(2):550-5.

Page $12 / 15$ 
25. Haacke EM, Xu Y, Cheng YC, Reichenbach JR. Susceptibility weighted imaging (SWI). Magnetic resonance in medicine. 2004;52(3):612-8.

26. Sehgal V, Delproposto Z, Haacke EM, Tong KA, Wycliffe N, Kido DK, Xu Y, Neelavalli J, Haddar D, Reichenbach JR. Clinical applications of neuroimaging with susceptibility-weighted imaging. Journal of magnetic resonance imaging. 2005;22(4):439-50.

27. Mittal S, Wu Z, Neelavalli J, Haacke EM. Susceptibility-weighted imaging: technical aspects and clinical applications, part 2. AJNR Am J Neuroradiol. 2009;30(2):232-52.

28. Haacke EM, Mittal S, Wu Z, Neelavalli J, Cheng YC. Susceptibility-weighted imaging: technical aspects and clinical applications, part 1. AJNR Am J Neuroradiol. 2009;30(1):19-30.

29. Jeerakathil T, Wolf PA, Beiser A, Massaro J, Seshadri S, D'Agostino RB, DeCarli C. Stroke risk profile predicts white matter hyperintensity volume: the Framingham Study. Stroke. 2004;35(8):1857-61.

30. Naka H, Nomura E, Wakabayashi S, Kajikawa H, Kohriyama T, Mimori Y, Nakamura S, Matsumoto M. Frequency of asymptomatic microbleeds on T2*-weighted MR images of patients with recurrent stroke: association with combination of stroke subtypes and leukoaraiosis. AJNR Am J Neuroradiol. 2004;25(5):714-9.

31. Gao T, Wang Y, Zhang Z. Silent cerebral microbleeds on susceptibility-weighted imaging of patients with ischemic stroke and leukoaraiosis. Neurological research. 2008;30(3):272-6.

32. Lee SH, Park JM, Kwon SJ, Kim H, Kim YH, Roh JK, Yoon BW. Left ventricular hypertrophy is associated with cerebral microbleeds in hypertensive patients. Neurology. 2004;63(1):16-21.

33. Masuda J, Tanaka K, Ueda K, Omae T. Autopsy study of incidence and distribution of cerebral amyloid angiopathy in Hisayama, Japan. Stroke. 1988;19(2):205-10.

34. Tsushima Y, Aoki J, Endo K. Brain microhemorrhages detected on T2*-weighted gradient-echo MR images. AJNR Am J Neuroradiol. 2003;24(1):88-96.

35. Seo SW, Hwa Lee B, Kim EJ, Chin J, Sun Cho Y, Yoon U, Na DL. Clinical significance of microbleeds in subcortical vascular dementia. Stroke. 2007;38(6):1949-51.

\section{Figures}



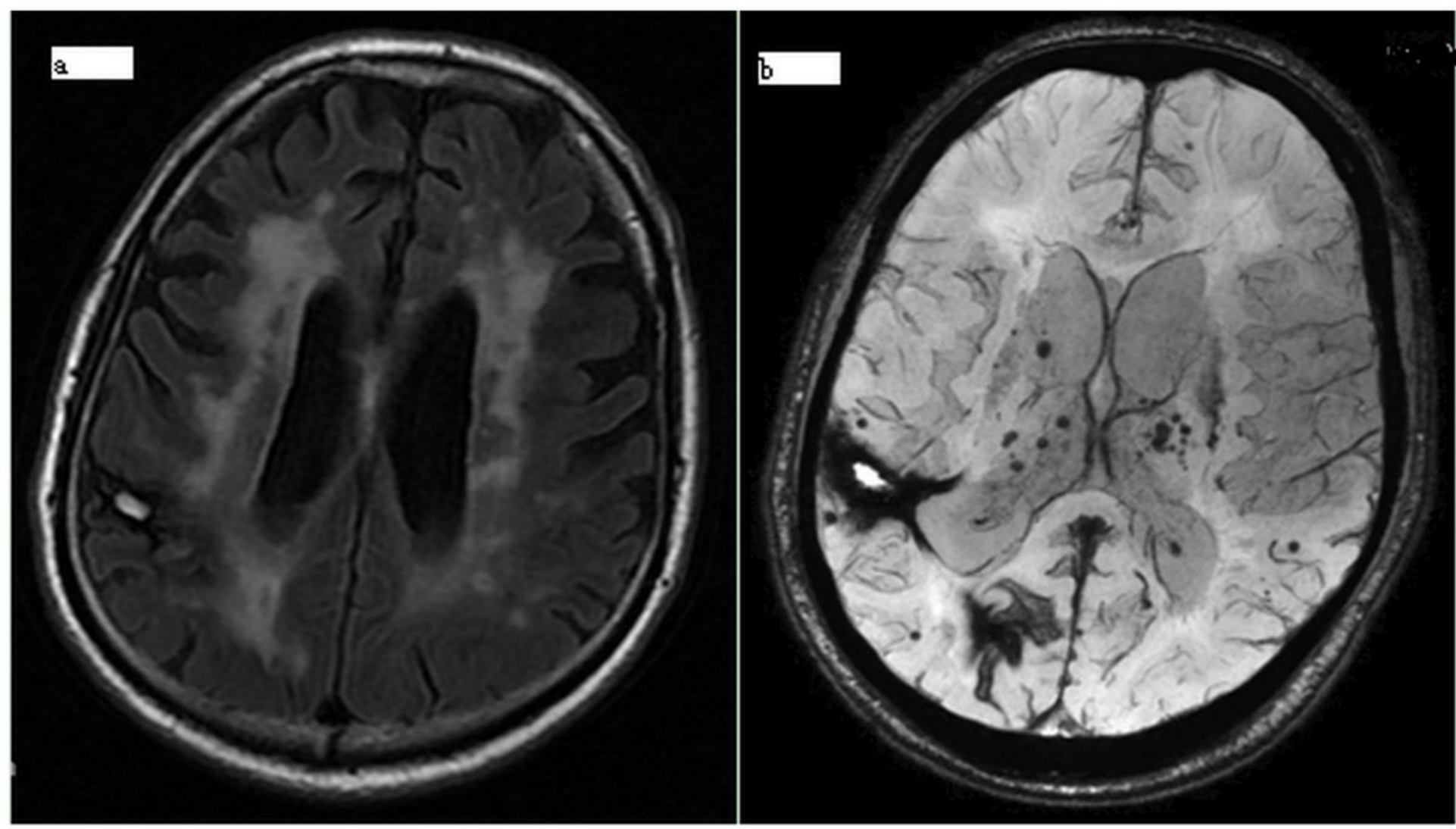

Figure 1

A 68-year-old male with stage 2 hypertension. (a) Axial T2 FLAIR MR image showing white matter lesions in the entire region around the lateral ventricle. The patient was scored as WMC grade 3. (b) An axial ESWAN MR image showing multiple CMBs in both cerebral hemispheres. The CMB degree was "severe."
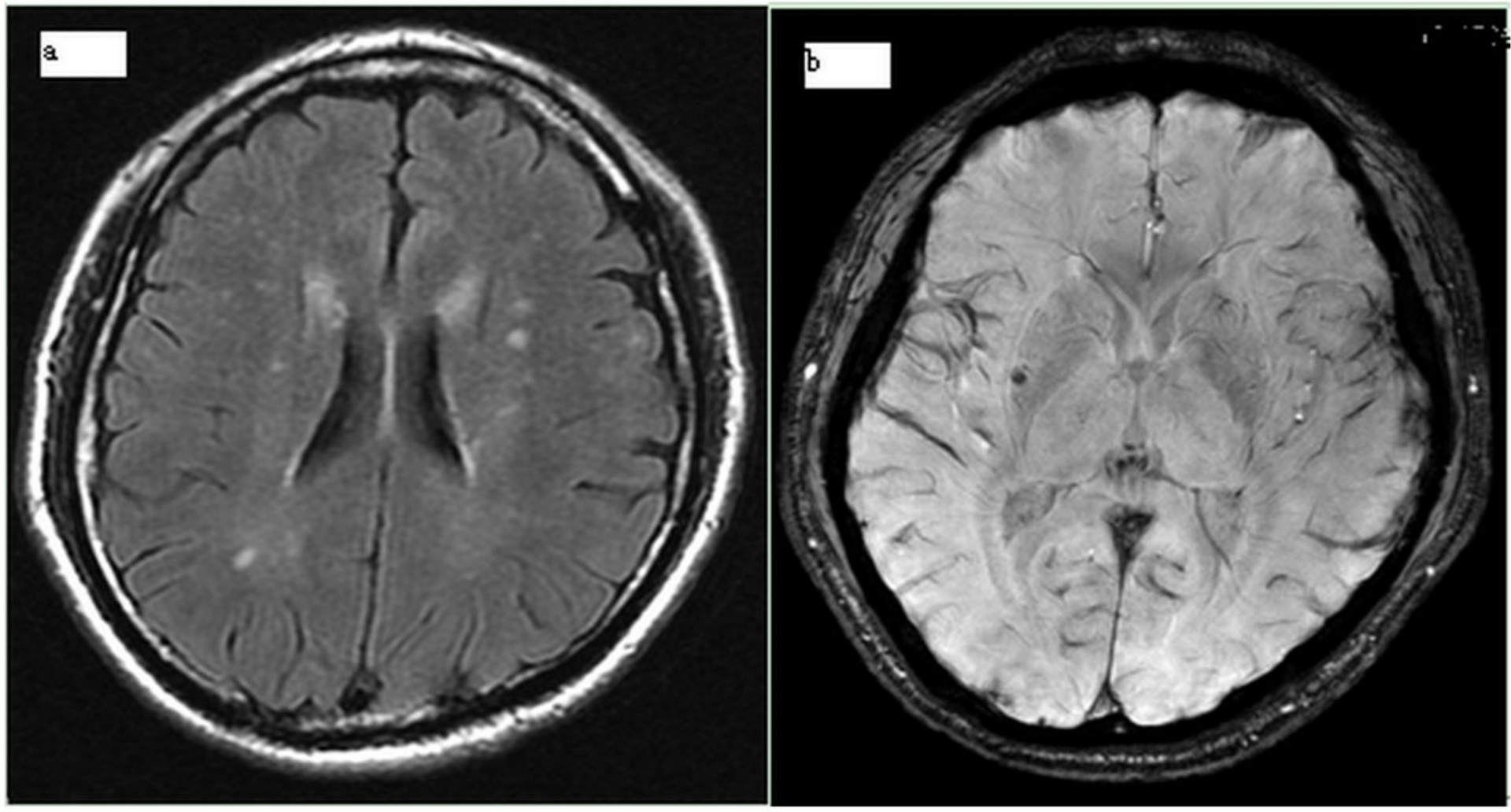


\section{Figure 2}

A 58-year-old female with stage 2 hypertension. (a) An axial T2 FLAIR MR image showing the beginning confluence of lesions around the lateral ventricle. The patient was scored as WMC grade 2. (b) An axial ESWAN MR image showing a single CMB in the right basal ganglia. The CMB degree was "mild." 